\title{
Hestia criteria can discriminate high- from low-risk patients with pulmonary embolism
}

\author{
Wendy Zondag*, Birgitta I. Hiddinga*, Monique J.T. Crobach ${ }^{\mp}$, Geert Labots ${ }^{+}$, \\ Anneke Dolsma ${ }^{\xi}$, Marc Durian ${ }^{f}$, Laura M. Faber**, Herman M.A. Hofstee ${ }^{\# \#,}$ \\ Christian F. Melissant ${ }^{\top \uparrow}$, Eric F. Ullmann ${ }^{++}$, Lies M.A. Vingerhoets ${ }^{\S \S}$, \\ Mariëlle J.M. de Vreede ${ }^{f f}$ and Menno V. Huisman* on behalf of The Hestia Study \\ Investigators***
}

ABSTRACT: We investigated whether the clinical criteria used in the Hestia study for selection of pulmonary embolism (PE) patients for outpatient treatment could discriminate PE patients with high and low risk for adverse clinical outcome.

We performed a cohort study with PE patients who were triaged with 11 criteria for outpatient treatment. Patients not eligible for outpatient treatment were treated in hospital. Study outcomes were recurrent venous thromboembolism, major bleeding and all-cause mortality during 3 months.

In total, $\mathbf{5 3 0}$ patients were included, of which $\mathbf{2 9 7}$ were treated at home. In the outpatient group, six patients $(2.0 \%, 95 \% \mathrm{Cl} 0.7-4.3 \%)$ had recurrent venous thromboembolism versus nine inpatients $(3.9 \%, 95 \% \mathrm{Cl} 1.9-7.0 \%)$. Three patients $(1.0 \%, 95 \% \mathrm{Cl} 0.2-2.9)$ died during the 3-months follow-up in the outpatient group versus 22 patients $(9.6 \%, 95 \% \mathrm{Cl} 6.3-14)$ in the in-patient group $(p<0.05)$. None of the outpatients died as a result of fatal PE versus five $(2.2 \%)$ in-patients $(\mathrm{p}<0.05)$. In the outpatient group, $0.7 \%(95 \% \mathrm{Cl} \mathbf{0 . 0 8 - 2 . 4 )}$ had major bleeding events versus $4.8 \%$ (95\% Cl 2.4-8.4) of in-patients $(p<0.05)$.

This study showed that the Hestia criteria can discriminate PE patients with low risk from patients with high risk for adverse clinical outcome. The low-risk patients can safely be treated at home.

KEYWORDS: Home treatment, mortality, outpatient treatment, venous thromboembolism

$\mathbf{N}$ owadays, most patients with acute pulmonary embolism (PE) start anticoagulant treatment in the hospital, but evidence on the safety of initial outpatient treatment in patients with PE is accumulating [1]. Two systematic reviews summarised the results of a few small observational studies on outpatient treatment in patients with PE $[2,3]$. These reviews concluded that although the evidence is not of high quality, it indicates that certain subgroups of patients with PE could be eligible for outpatient treatment. In 2010, two large retrospective studies on outpatient treatment of PE patients were published $[4,5]$. In these studies, low rates of adverse clinical outcome were reported, suggesting safety of outpatient treatment in $\mathrm{PE}$; in both articles, prospective validation of the results is recommended.

We have recently published the results of a large prospective study in which clinical signs and symptoms were used to select patients with PE for outpatient treatment [6]. The Hestia criteria consist of 11 clinical criteria that can be used as a bedside test. The purpose of the Hestia study was to evaluate the safety of outpatient treatment in patients with acute PE triaged by simple and easily performed Hestia criteria. However, in the Hestia study, these selection criteria for outpatient treatment were used for the first time. The criteria have not yet been validated in other cohorts. In order to underline the discriminative power of the Hestia criteria, we wanted to show the contrast between the rates of adverse events in patients treated at home versus patients treated in the hospital. Therefore, the aim of the present extension of the Hestia study was to show the difference in adverse clinical outcome between high-risk patients, initially treated as in-patients, and low-risk patients, initially treated at home.

\section{PATIENTS AND METHODS}

\section{Overview}

The Hestia study is a multicentre prospective cohort study in patients with acute PE who were

\section{AFFILIATIONS}

*Dept of Thrombosis and Haemostasis, Leiden University Medical Centre,

${ }^{\S}$ Dept of Pulmonology, Diaconessenhuis, Leiden, \#Dept of Pulmonology, Medical Spectrum Twente, Enschede, 'Dept of General Internal Medicine, Bronovo Hospital,

${ }^{+}$Dept of General Internal Medicine, HAGA Hospital,

${ }^{f}$ Dept of General Internal Medicine, Haaglanden Medical Centre, The Hague,

${ }^{f}$ Dept of Hematology, Erasmus Medical Centre, Rotterdam,

**Dept of General Internal Medicine, Rode Kruis Hospital, Beverwijk,

\#\# Dept of Internal Medicine, VU University Medical Center, Amsterdam,

- Dept of Pulmonology, Spaarne Hospital, Hoofddorp

${ }^{++}$Dept of Pulmonology, Rijnstate Hospital, Arnhem, and

${ }^{\S \S}$ Dept of General Internal Medicine, Rijnland Hospital, Leiderdorp, The Netherlands.

${ }^{* * *} A$ list of all investigators is given in the Acknowledgements section.

CORRESPONDENCE

W. Zondag, Dept of Thrombosis and Haemostasis C4-70, Leiden University Medical Centre, Postbus 9600, 2300 RC Leiden, The Netherlands

E-mail: W.Zondag@lumc.nl

Received:

Feb 212012

Accepted after revision:

June 012012

First published online:

July 122012

European Respiratory Journal

Print ISSN 0903-1936

Online ISSN 1399-3003 
selected for outpatient treatment using the Hestia criteria. The methods of this study are described elsewhere [6].

In the Hestia study, we prospectively registered all patients that were excluded from outpatient treatment and the reasons why they were excluded. The excluded patients were not included in our study because they were neither eligible for the intervention of outpatient treatment nor were they followed prospectively.

For the analysis described in this article, we retrospectively reviewed the medical charts of the patients excluded from home treatment to investigate whether they had a recurrent venous thromboembolism (VTE), bleeding or died 3 months following the PE. All suspected outcome events were classified by an independent central adjudication committee whose members were not participating in the study. This was the same committee as used for the initial Hestia study. The Hestia study protocol was approved by the institutional review board of each participating hospital.

\section{Patients}

Consecutive patients were included according to the following inclusion criteria: age $>18$ yrs with proven acute PE presenting to the emergency department. Patients with asymptomatic or chronic PE were not included.

Patients were admitted to the hospital if one of the following exclusion criteria (the Hestia criteria) for outpatient treatment were fulfilled: haemodynamic instability; thrombolytic treatment or embolectomy; high risk of bleeding; oxygen therapy; intravenous pain medication; diagnosis of PE while on therapeutic anticoagulant treatment; medical or social condition necessitating hospital admission; renal or liver impairment; pregnancy or history of heparin-induced thrombocytopenia.

This checklist with 11 items can be used as bedside test and can be completed within $5 \mathrm{~min}$. If none of the items was present, the patient was treated at home. All patients were treated with standard anticoagulant therapy according to international guidelines [7].

\section{Outcome events}

Symptomatic recurrent VTE was the main efficacy parameter and was considered present if recurrent PE or DVT were documented objectively, or, in the case of death, in which PE was demonstrated by autopsy or could not be confidently ruled out as a contributory cause. Major bleeding was the main safety outcome and was defined according to international guidelines [8]. Mortality was defined as death due to recurrent PE (fatal PE), fatal bleeding, cancer, or another established diagnosis. Information about the cause of death was obtained from either an autopsy report or a clinical report.

\section{Statistical analysis}

The power calculation of the Hestia study is described elsewhere [6]. In the initial Hestia study, we screened patients for eligibility for outpatient treatment until we reached our calculated sample size of patients treated at home. The group of PE patients treated in the hospital, described in this article, consists of consecutive patients who could not be treated at home. These patients were prospectively collected in all 12 hospitals participating in the Hestia study from May 2008 until April 2010.
Differences in baseline characteristics and outcome between the in- and outpatient groups were measured using Fisher's exact test for categorical variables and with an unpaired t-test for continuous variables. SPSS software version 17.0 (SPSS Inc, Chicago, IL, USA) was used for all analyses.

\section{RESULTS}

Between 2008 and 2010, 581 patients with acute, symptomatic PE presented to 12 Dutch hospitals (Leiden University Medical Centre, and Diaconessenhuis, Leiden; Medical Spectrum Twente, Enschede; Bronovo Hospital, Haga Hospital and Haaglanden Medical Centre, The Hague; Erasmus Medical Centre, Rotterdam; Rode Kruis Hospital, Beverwijk; VU University Medical Center, Amsterdam; Spaarne Hospital, Hoofddorp; Rijnstate Hospital, Arnhem; and Rijnland Hospital, Leiderdorp, all the Netherlands). Of these patients, 338 patients were potentially eligible for outpatient treatment; however, 41 patients were excluded for study reasons (e.g. refusal to participate or previous participation), leaving 297 patients for home treatment. In total, 243 patients were admitted to the hospital for the following reasons: haemodynamic instability $(n=30)$, thrombolytic treatment for massive $\mathrm{PE}(\mathrm{n}=5)$, high risk of bleeding $(\mathrm{n}=14)$, requirement of oxygen therapy $(n=73)$, severe pain requiring intravenous medication $(n=15)$, diagnosis of PE during anticoagulant treatment $(n=9)$, and either a medical $(n=63)$ or social $(n=24)$ condition necessitating admission to the hospital. In 10 patients, the reason for exclusion from outpatient treatment was not specified. 10 out of 243 patients treated in the hospital had to be excluded from this analysis, because the chart review revealed that pulmonary embolism was not objectively proven by imaging. This resulted in a total of 530 PE patients: 297 patients were treated as outpatients and 233 patients were treated in the hospital. The baseline characteristics of the outpatient and in-patient groups are shown in table 1. Patients treated in the hospital were significantly older, were more often immobilised and had more comorbidities (e.g. cancer, heart failure and chronic obstructive pulmonary disease) than patients treated at home. Four patients were lost to follow-up after hospital admission because they lived abroad.

\section{Outcome events}

\section{Recurrent VTE}

In patients treated at home, six $(2.0 \%, 95 \%$ CI $0.7-4.3 \%)$ had recurrent VTE; five patients had nonfatal recurrent PE and one patient had recurrent deep vein thrombosis (DVT). In patients treated in the hospital, nine $(3.9 \%, 95 \%$ CI $1.9-7.0 \%)$ had recurrent VTE; all patients had recurrent PE. More than half of all recurrent VTEs happened in the first 2 weeks after the initial PE (fig. 1). None of the outpatients had fatal PE, while five patients $(2.2 \%, 95 \%$ CI $0.8-4.8 \%)$ treated in the hospital died of fatal PE on day 1, 3, 6, 33 and 66 ( $p<0.05$; table 2). All three patients with fatal PE during the first week after the initial PE died during hospital admission. None of the fatal recurrences underwent autopsy to prove cause of death.

\section{Major bleeding}

Two outpatients had a major bleeding event $(0.7 \%, 95 \%$ CI $0.082-2.4 \%$; table 2$)$ versus 11 in-patients $(4.8 \%, 95 \%$ CI $2.4-8.4 \%$; $\mathrm{p}<0.05)$. The two major bleedings in the outpatients consisted of one fatal intracranial bleeding at day 7 and one large abdominal 


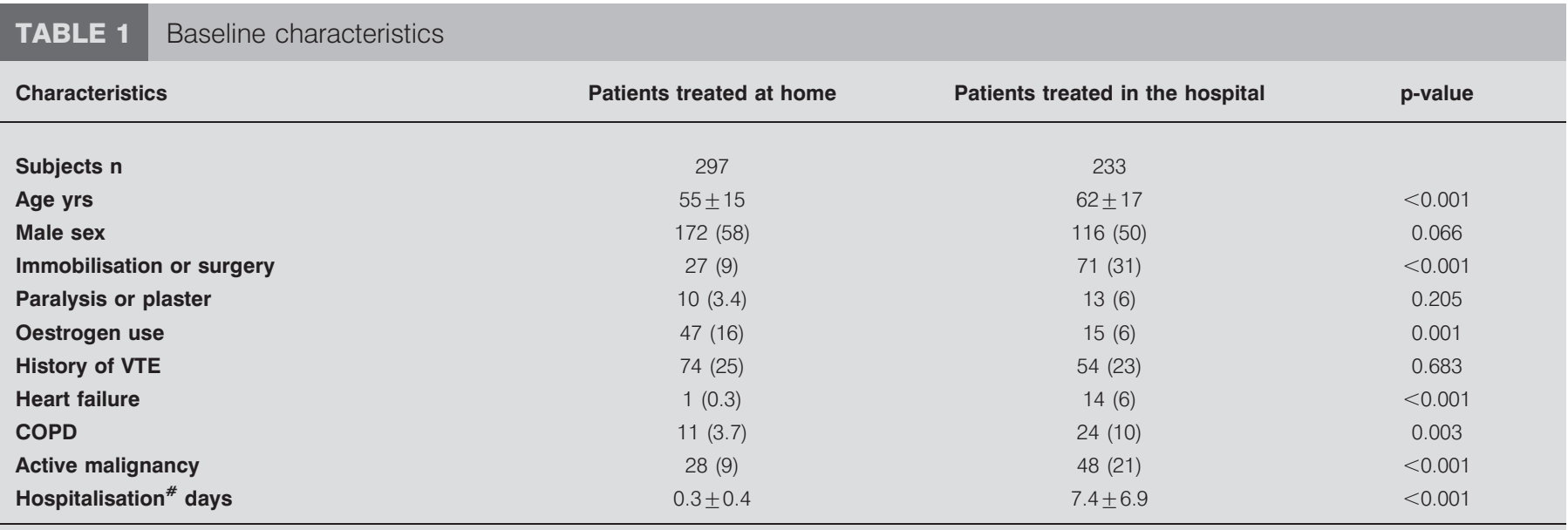

Data are presented as mean \pm SD or $n(\%)$, unless otherwise stated. VTE: venous thromboembolism; COPD: chronic obstructive pulmonary disease. ${ }^{\#}$ : data were missing in $126(23 \%)$ patients.

muscle haematoma at day 14 . Seven $(64 \%)$ of the major bleedings in the in-patient group happened during the first week of treatment (fig. 2). The locations of the 11 major bleedings in the in-patient group were: intracranial haemorrhage (fatal), two intra-abdominal bleedings, gastrointestinal bleeding, pericardial bleeding, bleeding in a pacemaker pocket, haemarthros, haematuria, large subcutaneous haematoma of arm and breast, intravenous catheter-related bleeding and a muscle haematoma of the upper leg. Five (45\%) out of 11 major bleedings in the in-patient group occurred during thrombolytic treatment, but none of these was fatal.

\section{Mortality}

During 3-months follow-up, 25 (4.8\%, 95\% CI 2.6-8.2\%) patients died. Seven (28\%) patients died of causes related to PE or bleeding, as described above. Other causes of death were mostly malignancies (nine patients, 36\%), respiratory insufficiency (five, 20\%) or myocardial infarction (two, $8 \%$ ). In the patients treated at home, three $(1.0 \%, 95 \%$ CI $0.21-2.9 \%)$ patients died versus $22(9.6 \%, 95 \%$ CI $6.3-14 \%)$ patients treated in the hospital $(\mathrm{p}<0.05$; table 2$)$. None of the patients treated at

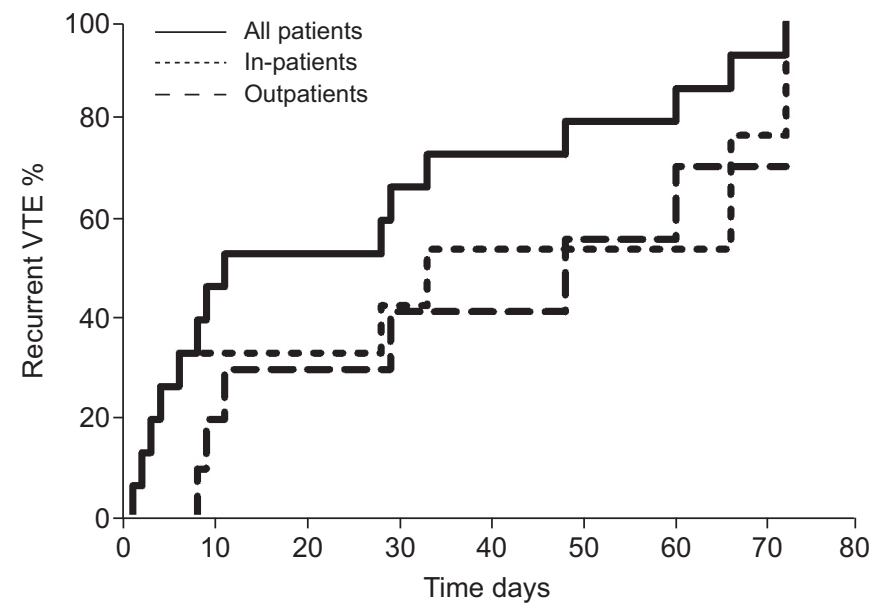

FIGURE 1. Timing of recurrent venous thromboembolism (VTE). home died within the first week versus four patients treated in the hospital $(p<0.05)$. Three of these in-patients died of fatal progression of PE and one in-patient had a fatal bleeding. Active malignancy was present in $16(64 \%)$ patients when they died. When patients with malignancies were excluded, 3month mortality was $0.4 \%$ (95\% CI $0.009-2.1)$ in the outpatients and $4.4 \%(95 \%$ CI $1.9-8.5)$ in the in-patients.

\section{DISCUSSION}

Our study demonstrates that when patients were selected for out- or in-patient treatment using the Hestia criteria, outpatients had fewer clinical adverse events than patients treated in the hospital. None of the outpatients died of fatal PE versus five of the in-patients; in-patients also had a higher overall mortality within the first week, and this period equals the average duration of hospital admission [9]. From this we can conclude that the Hestia criteria discriminated well between PE patients at high and low risk for adverse events and adequately selected low-risk patients for outpatient treatment.

In 2010, two retrospective studies on outpatient treatment of PE were published $[4,5]$. The Hestia study is an important prospective validation of these retrospective studies. Our results provide a firm validation and an extension of the results of the retrospective studies regarding the safety of outpatient treatment. In both the study presented here and the study of ERKENS et al. [4], the rate of fatal PE was $0 \%$ in the outpatient and $2 \%$ in the in-patient group. Overall, recurrent VTE rates, major bleeding rates and mortality are higher in the retrospective study than in the Hestia study, although not statistically because the confidence intervals overlap. In the Hestia study, we found recurrent VTE rates of $2.0 \%$ in the outpatients and $3.9 \%$ in the in-patients versus $3.8 \%$ in outpatients and $4.7 \%$ in in-patients in the retrospective study. Major bleeding rates were $0.7 \%$ in outpatients and $4.8 \%$ in inpatients in the Hestia study, and, in the study by ERKENS et al. [4], $1.5 \%$ and $6.1 \%$. Mortality was $5 \%$ in outpatients and $26 \%$ in in-patients in the study of ERKENS et al. [4], which is higher than in the $1.0 \%$ and $9.6 \%$, respectively, in the Hestia study. The explanation for the higher rates of adverse outcome in the retrospective study could be that their study population 
TABLE 2 Adverse clinical outcome in the 3-month follow-up period

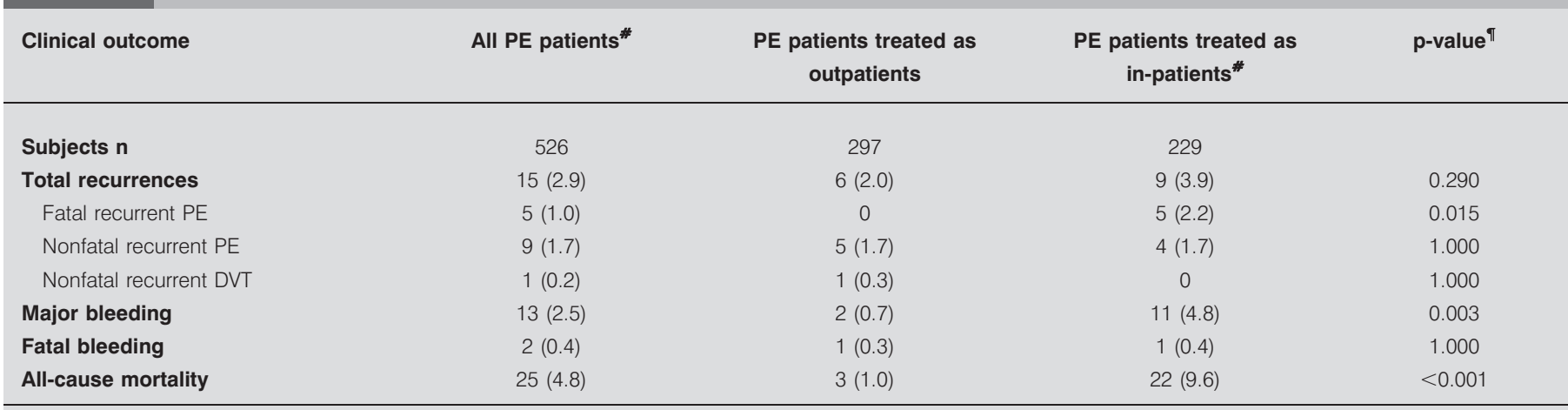

Data are presented as n (\%), unless otherwise stated. PE: pulmonary embolism; DVT: deep vein thrombosis. * : four in-patients were lost-to-follow-up; ": in-patients versus outpatients.

contained a higher proportion of patients with malignancies compared with the Hestia population ( $36 \%$ versus $14 \%$ ). The proportion of malignancies of $14 \%$ we found in the Hestia study is more comparable with proportions of malignancies observed in other large studies on anticoagulant treatment in patients with PE $[10,11]$. In our view, this adds to the generalisability of our results.

The other retrospective study by Kovacs et al. [5] did not give information on the clinical outcomes of patients treated in the hospital. The rates of adverse clinical outcome in the outpatient group are comparable with the rates in the Hestia study: none of the patients treated at home died of fatal PE.

Recently, the first randomised controlled trial on outpatient treatment in patients with PE was published [12]. They concluded that outpatient treatment was noninferior to inpatient treatment regarding recurrent VTE and mortality, but the major bleeding rate was a little higher in the outpatient group. The recurrent VTE and mortality rates in the outpatient group of the randomised trial were lower than the rates in the Hestia study, but this could be due to a highly selected population of young and healthy PE patients: mortality $0.6 \%$ and recurrent VTE $0.6 \%$. Despite the selection of young

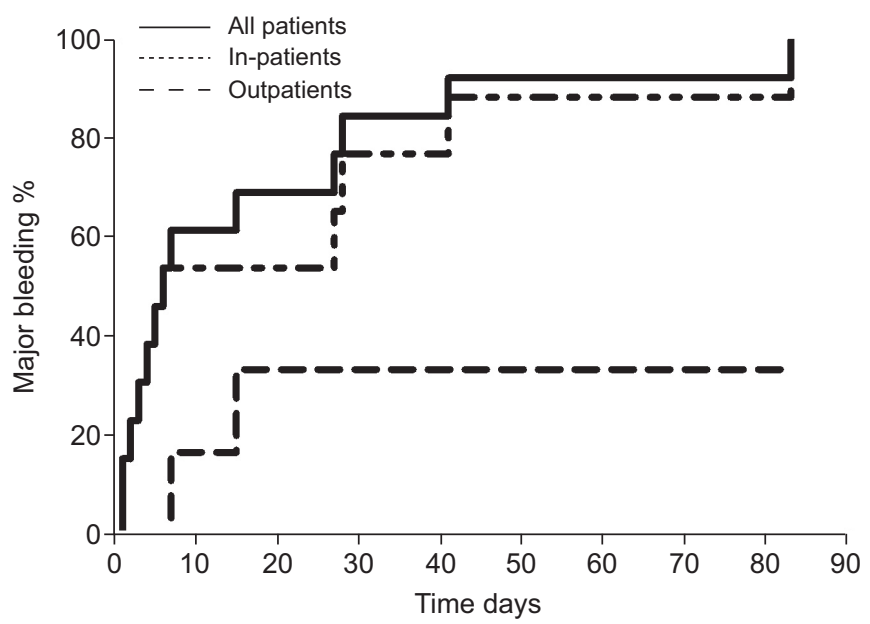

FIGURE 2. Timing of major bleeding patients with a low proportion of comorbidities, the major bleeding rate of $1.8 \%$ was higher than in our study, although the confidence intervals overlap.

The strength of the Hestia study is that it is the largest study on outpatient treatment; however, there are some limitations to our study: because we performed chart review and no prospective study follow-up of the in-patients, some events could have been missed. Also, because almost all patients had a complete follow-up, it is unlikely that we missed important events like fatal PE or fatal bleeding. Within the setting of the Hestia study, PE patients who were treated at home were closely followed. Before outpatient treatment can become a standard of care, it is essential that close follow-up of PE patients treated at home can be guaranteed in everyday patient care, especially during the first week.

Another limitation is that one patient in the home treatment group died of fatal intracranial bleeding. The exclusion of patients with a high bleeding risk with the Hestia criteria led to a significantly lower bleeding rate in patients treated at home versus patients treated in the hospital $(0.7 \%$ versus $4.8 \%$; $\mathrm{p}=0.003)$. Despite this careful triaging procedure, one patient in the home treatment group died of major bleeding. That patient had poorly controlled hypertension as an additional risk factor for bleeding in retrospect. Therefore, physicians should be very careful in selecting patients for outpatient treatment, especially those with risk factors for major bleeding.

In the study presented here, the Hestia criteria have been used to select patients with PE for outpatient or in-patient treatment. Comparable criteria have been used in other studies abroad, but Dutch doctors used these criteria for the first time. In the Hestia study, the criteria were used by doctors with different specialties and levels of experience. Taken together with the favourable findings, this reinforces the feasibility of these criteria to be used by all kinds of specialists without restriction to thrombosis experts. However, because it was the first time that the Hestia criteria had been used, these results need to be confirmed in future studies.

In conclusion, evidence on the safety of outpatient treatment in low-risk patients with PE is accumulating. The Hestia criteria can be used to discriminate PE patients with low risk for 
adverse clinical outcome from patients with high risk for adverse clinical outcome. The low-risk patients can be safely treated at home.

\section{SUPPORT STATEMENT}

This study was partially supported by an unrestricted research grant from GlaxoSmithKline, the Netherlands BV. GlaxoSmithKline had no influence in designing and writing the protocol, collection of data, performing the analysis of the study results and writing of the manuscript.

\section{CLINICAL TRIAL}

This study is registered with the Netherlands Trial Register with identifier number 1319.

\section{STATEMENT OF INTEREST}

Statements of interest for M.V. Huisman and for the study itself can be found at www.erj.ersjournals.com/site/misc/statements.xhtml

\section{ACKNOWLEDGEMENTS}

In addition to the authors, the following investigators have participated in this study as part of the Hestia Study Group: T. Vlasveld (Bronovo Hospital, The Hague); M.J.H.A. Kruip (Erasmus Medical Centre, Rotterdam); R. Valentijn and D. Creemers-Schild (HAGA Medical Centre, The Hague); F.H. Heyning and M Sikkens (Haaglanden Medical Centre, The Hague); M. Eijsvogel (Medisch Spectrum Twente, Enschede); I.C.M. Mos (Leiden University Medical Centre, Leiden); G.J.P.M. Jonkers and M. Donker (Rijnland Hospital, Leiderdorp); M.M.C. Hovens and H.A.H. Kaasjager (Rijnstate Hospital, Arnhem); C.F. Melissant (Spaarne Hospital, Hoofddorp); and M.H.H. Kramer and J. Post (VU Medical Centre, Amsterdam, all the Netherlands).

The independent adjudication committee is as follows: $\mathrm{H}$. Ten Cate and K. Hamulyak (Maastricht University Medical Centre, Maastricht); and V. Gerdes (Slotervaart Hospital, Amsterdam, both the Netherlands).

\section{REFERENCES}

1 Kearon C, Akl EA, Comerota AJ, et al. Antithrombotic therapy for VTE disease: antithrombotic therapy and prevention of thrombosis, 9th edn. American College of Chest Physicians Evidence-Based Clinical Practice Guidelines. Chest 2012; 141: e419S-e494S.

2 Janjua M, Badshah A, Matta F, et al. Treatment of acute pulmonary embolism as outpatients or following early discharge. A systematic review. Thromb Haemost 2008; 100: 756-761.

3 Squizzato A, Galli M, Dentali F, et al. Outpatient treatment and early discharge of symptomatic pulmonary embolism: a systematic review. Eur Respir J 2009; 33: 1148-1155.

4 Erkens PM, Gandara E, Wells P, et al. Safety of outpatient treatment in acute pulmonary embolism. J Thromb Haemost 2010; 8: 2412-2417.

5 Kovacs MJ, Hawel JD, Rekman JF, et al. Ambulatory management of pulmonary embolism: a pragmatic evaluation. J Thromb Haemost 2010; 8: 2406-2411.

6 Zondag W, Mos IC, Creemers-Schild D, et al. Outpatient treatment in patients with acute pulmonary embolism: the Hestia Study. J Thromb Haemost 2011; 9: 1500-1507.

7 Kearon C, Kahn SR, Agnelli G, et al. Antithrombotic therapy for venous thromboembolic disease: American College of Chest Physicians Evidence-Based Clinical Practice Guidelines (8th edn). Chest 2008; 133: 454S-545S.

8 Schulman S, Kearon C. Definition of major bleeding in clinical investigations of antihemostatic medicinal products in nonsurgical patients. J Thromb Haemost 2005; 3: 692-694.

9 Aujesky D, Stone RA, Kim S, et al. Length of hospital stay and postdischarge mortality in patients with pulmonary embolism: a statewide perspective. Arch Intern Med 2008; 168: 706-712.

10 van BA, Buller HR, Huisman MV, et al. Effectiveness of managing suspected pulmonary embolism using an algorithm combining clinical probability, D-dimer testing, and computed tomography. JAMA 2006; 295: 172-179.

11 Simonneau G, Sors H, Charbonnier B, et al. A comparison of lowmolecular-weight heparin with unfractionated heparin for acute pulmonary embolism. The THESEE Study Group. Tinzaparine ou Heparine Standard: Evaluations dans l'Embolie Pulmonaire. N Engl J Med 1997; 337: 663-669.

12 Aujesky D, Roy PM, Verschuren F, et al. Outpatient versus inpatient treatment for patients with acute pulmonary embolism: an international, open-label, randomised, non-inferiority trial. Lancet 2011; 378: 41-48. 